\title{
Prognostic value of pulmonary hemodynamic parameters in cardiac transplant candidates
}

\author{
Małgorzata Sobieszczańska-Małek, Tomasz Zieliński, Walerian Piotrowski, \\ Jerzy Korewicki; on behalf of the participants of POLKARD-HF \\ Heart Failure and Transplantology Department, Institute of Cardiology, Warsaw, Poland
}

\begin{abstract}
Background: Pulmonary hypertension is a contradiction for heart transplantation (HTx). The aim of the study was to examine prognostic significance of pulmonary hemodynamic variables in patients with severe chronic heart failure (HF) considered for HTx.

Methods: Patients with HF were qualified to HTx in Poland. We measured pulmonary artery systolic pressure (PASP), pulmonary capillary wedge pressure (PCWP), transpulmonary gradient (TPG), cardiac output (CO), pulmonary vascular resistance (PVR) and systemic vascular resistance (SVR). We performed biochemical evaluation, 6-min walking test, $\mathrm{VO}_{2}$ max. Death or emergency HTx were assumed as the endpoints in the follow-up. Death or any kind of HTx were considered an end of observation. Survival analysis was conducted using Kaplan-Meier curves (long rank test with strait defined by terciles of analyzed hemodynamic parameters).
\end{abstract}

Results: Six hundred and fifty-eight patients were qualified to HTx between 2003 and 2007. The mean follow-up: 601 days. 87.8\% male. Mean age was under 50, III and IV NYHA class. Mean PASP was $44.3 \pm 16.9 \mathrm{~mm} \mathrm{Hg}$, TPG $10.0 \pm 6.6 \mathrm{~mm} \mathrm{Hg}$ and PVR $2.9 \mathrm{~mm} \mathrm{Hg}, P C W P$ $20.9 \pm 9.3 \mathrm{~mm} H \mathrm{Hg}$. PASP and PCWP had influence on survival or emergency HTx. There was a significant difference in survival between patients with $P C W P>25 \mathrm{~mm} \mathrm{Hg}$ and PCWP < $<25 \mathrm{~mm} \mathrm{Hg}$. The worst prognosis was with PASP higher than $50 \mathrm{~mm} \mathrm{Hg}$. One-, two-, and three-year survival was $75 \%, 58 \%$ and $48 \%$ compared to patients with PASP $<35 \mathrm{~mm} \mathrm{Hg}$ (80\%, $70 \%$, and $68 \%$, respectively).

Conclusions: In patients qualified to HTx, pathological values of pulmonary hemodynamic parameters have a significant influence on survival. The worst prognosis have patients with $P A S P>50 \mathrm{~mm} \mathrm{Hg}$, and PCWP > $25 \mathrm{~mm} \mathrm{Hg}$. Pulmonary hemodynamic parameters are important during allocation process to HTx. (Cardiol J 2014; 21, 5: 532-538)

Key words: pulmonary hypertension, heart transplantation

\section{Introduction}

Pulmonary pressure may be increased in patients with heart failure (HF). Pulmonary hypertension $(\mathrm{PH})$ occurs in 30-35\% of patients with $\mathrm{HF}$, and carries a poor prognosis [1].
In patients referred for heart transplantation (HTx), PH with pulmonary vascular resistance (PVR) above 3.5 Wood Units is reported in 20-35\% [2].

According to European Society of Cardiology (ESC), $\mathrm{PH}$ is defined as a mean pulmonary artery

Address for correspondence: Dr n. med. Małgorzata Sobieszczańska-Małek, Heart Failure and Transplantology Department, Institute of Cardiology, ul. Alpejska 42, 04-628 Warszawa, Poland, e-mail: m.sobieszczanska@ikard.pl 
pressure higher than $25 \mathrm{~mm} \mathrm{Hg}$ at rest and higher than $30 \mathrm{~mm} \mathrm{Hg}$ with exercise [3]. Taking into account that mean pulmonary capillary wedge pressure (PCWP) in patients with severe chronic HF exceeds $20-22 \mathrm{~mm} \mathrm{Hg}$, one could speculate that $\mathrm{PH}$ defined in accordance with ESC criteria may be diagnosed in about $80 \%$ of patients.

According to International Society for Heart and Lung Transplantation guidelines, fixed PH and some other hemodynamic parameters could be considered a relative contraindication for HTx [4]. Among these are: pulmonary artery systolic pressure $(\mathrm{PASP}) \geq 60 \mathrm{~mm} \mathrm{Hg}$, with PVR $>5 \mathrm{WU}$, or transpulmonary gradient (TPG) $16-20 \mathrm{~mm} \mathrm{Hg}$. The risk of right HF and early death after HTx is increased in this group of patients [5].

$\mathrm{PH}$ is a powerful predictor of mortality in $\mathrm{HF}$ and it is independent of other predictors such as: $\mathrm{N}$-terminal of the prohormone B-type natriuretic peptide (NT-proBNP), echo-variables, mitral regurgitation, and HF symptoms. That means that they should be taken into account during allocating (elective vs. urgent) patients for HTx [6].

Probably, the current recipient's allocation system - UNOS should be modified, taking into account special consideration for ambulatory patients qualified for HTx with high risk of death because of $\mathrm{PH}$.

Our main aim was to examine prognostic significance of pulmonary hemodynamic variables in homogenic group of patients with severe chronic HF considered for HTx.

\section{Methods}

\section{Study population and procedures}

Between November 12003 and October 31 2007 there were 658 patients with severe chronic HF qualified to HTx in 4 cardiac transplant centers in Poland. During diagnostic procedures, right heart catheterization was performed with the use of Swan-Ganz thermodilution catheter for evaluation of pulmonary hemodynamics. Cardiac output (CO), pulmonary artery pressure, PCWP, TPG, PVR and systemic vascular resistance (SVR) were measured and calculated. Among 658 patients registered, in 559 the whole protocol of pulmonary hemodynamic parameter was available. During the qualification to HTx other standard diagnostic procedures were performed: biochemical evaluation (sodium, NT-proBNP, high sensitivity-C-reactive protein [hs-CRP]), 6-min walking test, maximal oxygen uptake $\left(\mathrm{VO}_{2} \mathrm{max}\right)$. Heart Failure Survival Score (HFSS) was calculated according to Aaronson.
The average observation time was 601 days (1-1462). Death or emergency HTx were assumed as the endpoints in the follow-up. Death or any kind of HTx were considered censoring events.

The study was approved by the local bioethical committee and all patients gave their informed consent.

\section{Statistical analysis}

The following statistical methods were used: means, standard deviations, medians and terciles in distribution. Comparisons of continuous parameters were conducted using analysis of variance (Duncan test) which met the criteria for normal distribution. In case of other distribution, non-parametrical Wilcoxon or Kruskal-Wallis tests were used. For evaluation of survival, terciles of some pulmonary hemodynamic variables were compared. Kaplan-Meier statistics were used together with the log-rank test for verification of the hypothesis of homogeneity of the survival rate curves. Prediction value of the analyzed variables in terms of occurrence of the endpoint was analyzed using the Cox regression method with a single variable. Multifactor Cox regression model was built with the stepwise variable selection method. All analyses were performed using statistical package STATISTICA 10 and a p-value of $<0.05$ was considered statistically significant.

\section{Results}

Between November 12003 and October 31 2007, 658 patients were qualified to HTx in Poland and registered. In 559 (84.9\%) full hemodynamic protocol was obtained. The mean follow-up was 601 days. Most of qualified patients (87.8\%) were male. Mean age was 50.1 years. Most of them were in III and IV New York Heart Association (NYHA) class (84\%).

Echo assessment of left ventricular (LV) function demonstrated severe heart dysfunction: ejection fraction $20.7 \%$, LV end-diastolic diameter $72.2 \mathrm{~mm}$. Systolic blood pressure (SBP) was $100.9 \mathrm{~mm} \mathrm{Hg}$. Median levels of standard biomarkers were as follows: NT-proBNP $-2.703 \mathrm{pg} / \mathrm{mL}$, hs-CRP $-3.318 \mathrm{mg} / \mathrm{L}$. An Aaronson score (HFSS) equal to 7.61 indicated a moderate risk of death or emergency HTx.

Pulmonary hemodynamic parameters measured invasively revealed $\mathrm{PH}$ with PASP higher than $35 \mathrm{~mm} \mathrm{Hg}$ in 2/3 of patients. Mean PASP was $44.3 \pm 16.9 \mathrm{~mm} \mathrm{Hg}$, pulmonary gradient $10.1 \pm$ $\pm 5.6 \mathrm{~mm} \mathrm{Hg}$, and PVR $3.1 \pm 2.4$ Wood Unit. Mean value of PCWP was $21.0 \pm 9.5 \mathrm{~mm} \mathrm{Hg}$. 
Table 1. Baseline characteristic of all patients according to PASP terciles

\begin{tabular}{|c|c|c|c|}
\hline & PASP $<35$ mm Hg* & $35 \mathrm{~mm} \mathrm{Hg} \leq \mathrm{PASP}<50 \mathrm{~mm} \mathrm{Hg} *$ & PASP $\geq 50 \mathrm{~mm} \mathrm{Hg*}$ \\
\hline Age [years] & $52.6 \pm 8.6$ & $47.9 \pm 11.7$ & $48.6 \pm 11.7$ \\
\hline Weight [kg] & $78.2 \pm 13.6$ & $76.1 \pm 15.0$ & $78.8 \pm 15.9$ \\
\hline Height $[\mathrm{cm}]$ & $172.0 \pm 7.5$ & $173.2 \pm 9.4$ & $172.8 \pm 7.4$ \\
\hline LVEF [\%] & $21.7 \pm 7.9$ & $19.6 \pm 7.7$ & $19.5 \pm 6.6$ \\
\hline LVEDD [mm] & $72.2 \pm 10.6$ & $72.6 \pm 12.5$ & $72.7 \pm 11.4$ \\
\hline LVESD [mm] & $59.8 \pm 13.7$ & $62.9 \pm 12.7$ & $62.5 \pm 13.0$ \\
\hline Heart rate [/min] & $77.0 \pm 14.1$ & $79.3 \pm 16.0$ & $79.7 \pm 15.3$ \\
\hline Systolic BP [mm Hg] & $102.7 \pm 14.8$ & $100.2 \pm 13.5$ & $98.7 \pm 12.5$ \\
\hline Diastolic BP [mm Hg] & $66.8 \pm 11.0$ & $65.9 \pm 10.8$ & $64.5 \pm 8.8$ \\
\hline $\mathrm{Na}[\mathrm{mEq} / \mathrm{L}]$ & $136.6 \pm 4.3$ & $135.9 \pm 4.3$ & $135.6 \pm 4.8$ \\
\hline $\mathrm{VO}_{2 \max }$ & $11.9 \pm 3.3$ & $12.0 \pm 3.1$ & $12.0 \pm 3.0$ \\
\hline HFSS & $7.7 \pm 0.9$ & $7.6 \pm 0.8$ & $7.4 \pm 0.9$ \\
\hline NT-proBNP [pg/mL]* & $1705.0(1030.0-4130.0)$ & $3363.0(2055.0-5813.0)$ & $3501.0(1931.5-5423.5)$ \\
\hline hs-CRP $[\mathrm{mg} / \mathrm{L}]^{*}$ & $3.2(1.1-6.3)$ & $6.4(0.8-8.4)$ & $15.1(0.1-347.0)$ \\
\hline PASP $[\mathrm{mm} \mathrm{Hg}]$ & $26.6 \pm 6.0$ & $44.0 \pm 4.5$ & $63.4 \pm 11.2$ \\
\hline PCWP [mm Hg] & $12.3 \pm 5.5$ & $22.2 \pm 5.7$ & $29.4 \pm 7.7$ \\
\hline TPG [mm Hg] & $6.9 \pm 3.6$ & $8.8 \pm 3.6$ & $13.5 \pm 6.2$ \\
\hline Cardiac output [L/min] & $4.0 \pm 2.5$ & $3.7 \pm 1.2$ & $3.6 \pm 1.1$ \\
\hline PVR [Wood Units] & $2.0 \pm 0.9$ & $2.7 \pm 1.5$ & $4.1 \pm 2.6$ \\
\hline SVR [Wood Units] & $22.5 \pm 7.6$ & $23.1 \pm 8.4$ & $23.4 \pm 8.4$ \\
\hline PVR/SVR & $0.1 \pm 0.09$ & $0.1 \pm 0.08$ & $0.2 \pm 0.08$ \\
\hline PASP/Systolic BP & $0.26 \pm 0.08$ & $0.45 \pm 0.07$ & $0.65 \pm 0.14$ \\
\hline
\end{tabular}

*Mean \pm standard deviation, median (low-high quartile); LVEF — left ventricular ejection fraction; LVEDD — left ventricular end-diastolic diameter; LVESD - left ventricular end-systolic diameter; BP — blood pressure; Na — sodium serum concentration; HFSS - Heart Failure Survival Score; NT-proBNP - N-terminal of the prohormone B-type natriuretic peptide; hs-CRP — high sensitivity C-reactive protein;

PASP — pulmonary artery systolic pressure; PCWP — pulmonary capillary wedge pressure; TPG — total pulmonary gradient;

PVR - pulmonary vascular resistance; SVR — systemic vascular resistance

All patients were treated with optimally tolerated doses of angiotensin converting enzyme inhibitors and/or angiotensin II receptor blockers $-90 \%$ of patients (doses: ramipril $5.50 \pm 4.32$, perindopril $3.63 \pm 1.63$, enalapril $15.83 \pm 10.88$ $\mathrm{mg} / \mathrm{d}$.). Beta-blockers $-91 \%$ of patients (doses: carvedilol $17.23 \pm 13.64 \mathrm{mg} / \mathrm{d}$., bisoprolol $3.59 \pm$ $\pm 2.79 \mathrm{mg} / \mathrm{d}$.), diuretics $-93 \%$ of patients, digoxin $-44 \%$ of patients, mineralocorticoid receptor antagonists $-78 \%$ of patients by cardiologists in $\mathrm{HF}$ or transplantology departments and appropriate outpatient clinics.

Out of 559 patients qualified to HTx $24.9 \%$ died or underwent emergency HTx during the follow-up. Baseline characteristics of all patients according to PASP terciles — see Table 1.

\section{Prognostic value of pulmonary hemodynamic parameters}

Among 21 analyzed parameters (variables) age, ejection fraction [\%], SBP and diastolic blood pressure (DBP), LV end-systolic diameter, sodium serum level, Aaronson risk score (HFSS), NT-
-proBNP and hs-CRP levels, PCWP, and PASP/ /SBP ratio, had influence on patients' survival in univariate Cox regression analysis (Table 2). Multifactor Cox regression model revealed that only sodium serum level, NT-proBNP, and hs-CRP levels significantly influenced survival of patients qualified to HTx (Table 3).

The main aim of the study was to examine prognostic significance of pulmonary hemodynamic variables of patients with severe chronic HF qualified to HTx. Among all hemodynamic parameters recorded during Swan-Ganz catheterization SBP, PCWP, and PASP/SBP score had statistically significant influence on patients' survival in univariate Cox regression model (Table 4). Some variables (SBP, PCWP, and PASP/ /SBP) had influence on survival or emergency HTx with the use of multifactor Cox regression model (Table 5).

For the outcome analysis Kaplan-Meier survival curves were generated and the differences between terciles of PCWP, PASP, SBP, and PASP/ /SBP score were assessed using the log-rank test. 
Table 2. Univariate Cox regression analyses of factors influencing survival of cardiac transplant candidates.

\begin{tabular}{lcc}
\hline Parameters & $\begin{array}{c}\text { HR estimates } \\
\text { [univariate] }\end{array}$ & $\begin{array}{c}-95 \% \mathrm{Cl}- \\
+95 \% \mathrm{Cl}\end{array}$ \\
\hline Age [years] & $0.984^{*}$ & $0.968-0.999$ \\
Weight [kg] & 0.997 & $0.985-1.009$ \\
LVEF [\%] & $0.948^{*}$ & $0.921-0.997$ \\
LVEDD [mm] & 1.016 & $0.998-1.034$ \\
LVESD [mm] & $1.018^{*}$ & $1.003-1.034$ \\
Heart rate [/min] & $1.018^{*}$ & $1.008-1.029$ \\
Systolic BP [mm Hg] & $0.981^{*}$ & $0.968-0.994$ \\
Diastolic BP [mm Hg] & $0.982^{*}$ & $0.965-0.999$ \\
Na [mEq/L] & $0.925^{*}$ & $0.893-0.957$ \\
HFSS & $0.565^{*}$ & $0.468-0.687$ \\
NT-proBNP [pg/mL] & $1.007^{*}$ & $1.005-1.009$ \\
hs-CRP [mg/L] & $1.011^{*}$ & $1.006-1.015$ \\
PASP [mm Hg] & 1.008 & $0.998-1.018$ \\
PCWP [mm Hg] & $1.030^{*}$ & $1.013-1.048$ \\
TPG [mm Hg] & 0.995 & $0.966-1.025$ \\
Cardiac output [L/min] & 0.923 & $0.789-1.080$ \\
PVR [Wood Units] & 0.990 & $0.927-1.058$ \\
SVR [Wood Units] & 1.013 & $0.991-3.116$ \\
PVR/SVR & 0.761 & $0.186-4.681$ \\
PASP/Systolic BP & $11.003^{*}$ & $3.126-38.726$ \\
\hline P O.05;HR & &
\end{tabular}

${ }^{*} \mathrm{p}<0.05$; HR - hazard ratio; $\mathrm{Cl}$ - confidence interval; rest abbreviations as in Table 1

Table 3. Multifactor Cox regression model built with stepwise variable selection method of factors influencing survival.

\begin{tabular}{lcc}
\hline Parameters & $\begin{array}{c}\text { HR estimates } \\
\text { [multifactor] }\end{array}$ & $\begin{array}{c}-95 \% \mathrm{Cl}- \\
+95 \% \mathrm{Cl}\end{array}$ \\
\hline hs-CRP & $1.008^{*}$ & $1.003-1.012$ \\
$\mathrm{Na}[\mathrm{mEq} / \mathrm{L}]$ & $0.927^{*}$ & $0.887-0.969$ \\
$\mathrm{NT}$-proBNP $[\mathrm{pg} / \mathrm{mL}]$ & $1.006^{*}$ & $1.003-1.009$ \\
\hline
\end{tabular}

* $\mathrm{p}<0.05 ; \mathrm{HR}$ - hazard ratio; $\mathrm{Cl}$ - confidence interval; rest abbreviations as in Table 1

There was a significant difference in survival between patients in whom PCWP was higher than $25 \mathrm{~mm} \mathrm{Hg}$ and those with PCWP $<25 \mathrm{~mm} \mathrm{Hg}$. No difference was found between those with PCWP 16-25 $\mathrm{mm} \mathrm{Hg}$ and those with PCWP $16 \mathrm{~mm} \mathrm{Hg}$ and lower (Fig. 1).

There was a significant difference as far as PASP was concerned. The worst prognosis had the patients with PASP above $50 \mathrm{~mm} \mathrm{Hg}-1-, 2-$, 3 -year survival was $75 \%, 58 \%$, and $48 \%$, as compared to patients with PASP $<35 \mathrm{~mm} \mathrm{Hg}(80 \%$, $70 \%$, and $68 \%$, respectively).
Table 4. Univariate Cox regression analysis of hemodynamic parameters influencing survival.

\begin{tabular}{lcc}
\hline Parameters & $\begin{array}{c}\text { HR estimates } \\
\text { [univariate] }\end{array}$ & $\begin{array}{c}-95 \% \mathrm{Cl}- \\
+95 \% \mathrm{Cl}\end{array}$ \\
\hline Systolic BP [mm Hg] & $0.981^{*}$ & $0.968-0.995$ \\
Diastolic BP [mm Hg] & 0.984 & $0.967-1.001$ \\
PASP [mm Hg] & 1.008 & $0.998-1.018$ \\
PCWP mean [mm Hg] & $1.030^{*}$ & $1.012-1.048$ \\
TPG [mm Hg] & 0.993 & $0.963-1.024$ \\
Cardiac output [L/min] & 0.916 & $0.779-1.078$ \\
PVR [Wood Units] & 1.002 & $0.936-1.074$ \\
SVR [Wood Units] & 1.014 & $0.992-1.036$ \\
PVR/SVR & 0.842 & $0.106-6.669$ \\
PASP/Systolic BP & $11.00^{*}$ & $3.126-38.726$ \\
\hline
\end{tabular}

${ }^{*} \mathrm{p}<0.05 ; \mathrm{HR}$ - hazard ratio; $\mathrm{Cl}$ - confidence interval; rest abbreviations as in Table 1

Table 5. Multifactor Cox regression model of arbitrary selection pulmonary hemodynamic parameters potentially influencing survival.

\begin{tabular}{lcc}
\hline Parameters & $\begin{array}{c}\text { HR estimates } \\
\text { [multifactor] }\end{array}$ & $\begin{array}{c}-95 \% \mathrm{Cl}- \\
+95 \% \mathrm{Cl}\end{array}$ \\
\hline Systolic BP [mm Hg] & $0.985^{*}$ & $0.972-0.999$ \\
PCWP mean [mm Hg] & $1.025^{*}$ & $1.007-1.040$ \\
PASP/Systolic BP & 5229.00 & $1.128-24.232$ \\
\hline
\end{tabular}

* $\mathrm{p}<0.05$; HR - hazard ratio; $\mathrm{Cl}$ - confidence interval; rest abbreviations as in Table 1

It is worth to note that there was no difference in 200 days of survival between the second and third tercile patients with PASP > $35 \mathrm{~mm} \mathrm{Hg}$. One can speculate if short-term survival is worst in patients qualified to HTx with any level of $\mathrm{PH}$ (Fig. 2). Also, systemic blood pressure had an important influence on survival. Patients with SBP equal or lower than $90 \mathrm{~mm} \mathrm{Hg}$ had 1-, 2-, and 3-year survival $66 \%, 53 \%$ and $48 \%$ (Fig. 3 ).

Because high PASP and low SBP had significant influence on survival in cardiac transplant candidates we assessed prognostic value of PASP/ /SBP score (Fig. 4), values more than 0.52 were connected with worst prognosis.

\section{Discussion}

Heart transplantation is currently the best method of treatment for patients with terminal HF. Unfortunately, HTx is available only to a part of those qualified. Usually patients are listed for transplantation only when it is presumed that they will live longer and function better after transplantation 


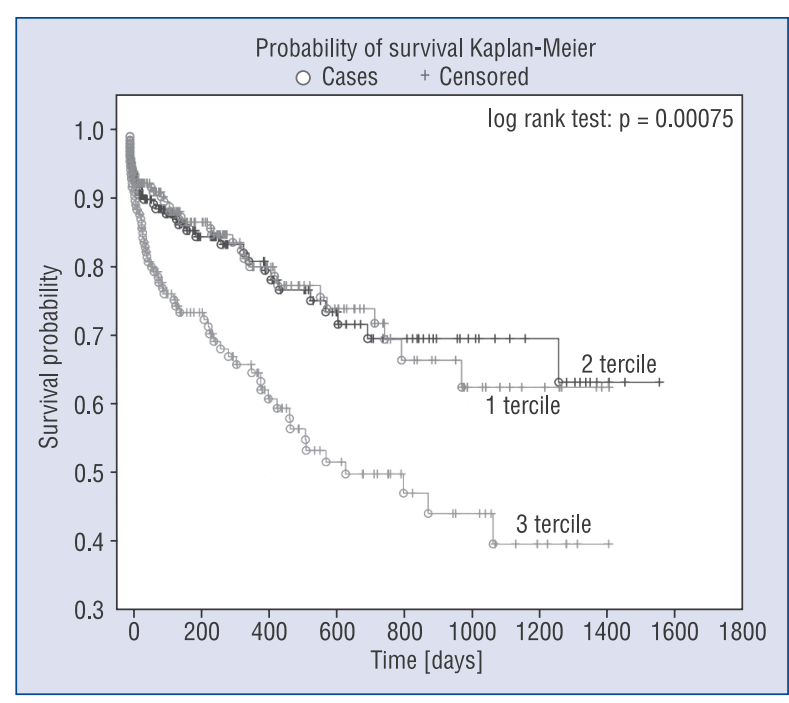

Figure 1. Probability of survival — patients stratified by pulmonary capillary wedge pressure; 1 tercile $\leq 16 \mathrm{~mm} \mathrm{Hg}$, 3 tercile $>25 \mathrm{~mm} \mathrm{Hg}$.

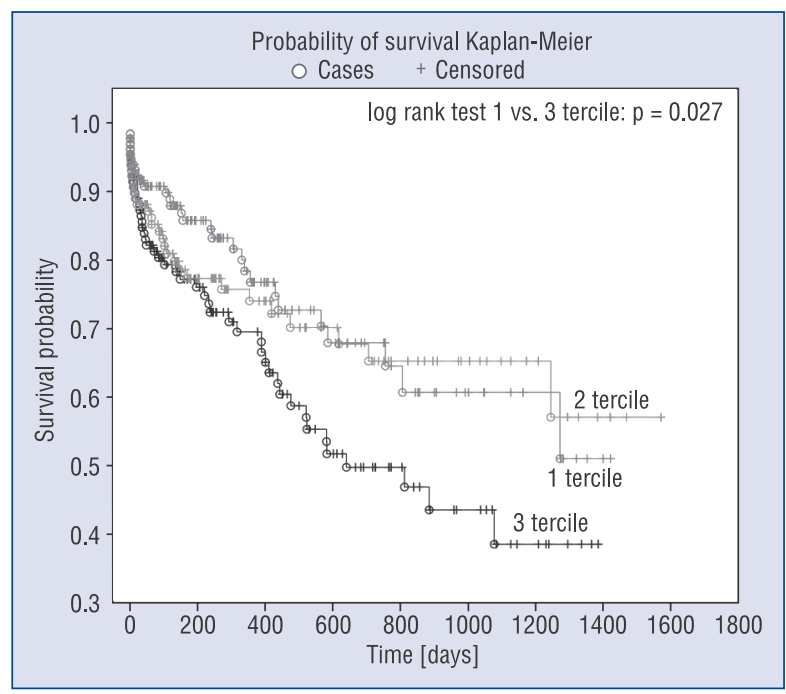

Figure 2. Probability of survival according to pulmonary artery systolic pressure; 1 tercile $\leq 35 \mathrm{~mm} \mathrm{Hg}$, 3 tercile $>50 \mathrm{~mm} \mathrm{Hg}$.

than on medical therapy. The main problem with patients listed for HTx is proper allocation. There is a shortage of donors leading to long waiting list, so organs should be allocated to the patients at high risk of dying, underscoring the importance of accurate prognosis determination.

The most widely accepted criteria for risk assessment are based on cardiopulmonary stress test $\left(\right.$ peakVO $\mathrm{VO}_{2}$ ) and HFSS. Patients with redu-

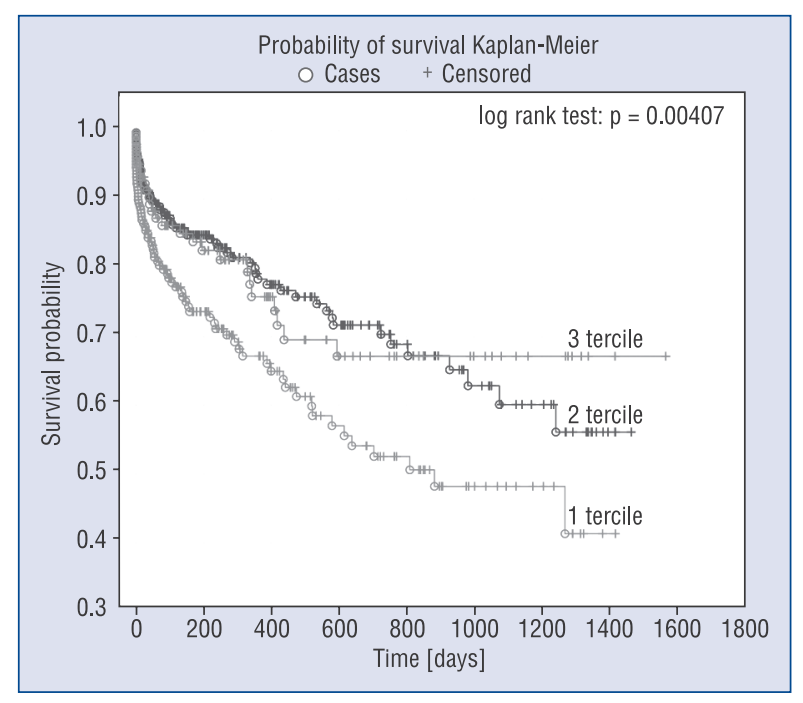

Figure 3. Probability of survival according to systolic blood pressure; 1 tercile $\leq 90 \mathrm{~mm} \mathrm{Hg}$, 3 tercile $>110 \mathrm{~mm} \mathrm{Hg}$.

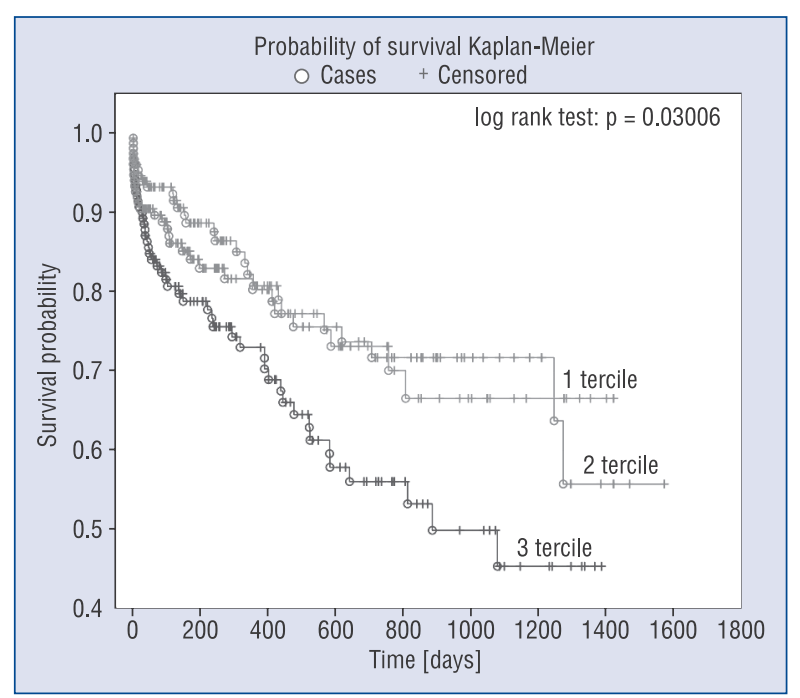

Figure 4. Probability of survival according to pulmonary artery systolic pressure vs. systolic blood pressure ratio; 1 tercile $\leq 0.36,3$ tercile $>0.52$.

ced capacity $\left(\mathrm{VO}_{2} \leq 14 \mathrm{~mL} / \mathrm{min} / \mathrm{kg}\right)$ had worse survival. HFSS this is a scoring system based on 7 parameters, including peak $\mathrm{VO}_{2}$, etiology of cardiomyopathy, resting heart rate, sodium level, LV ejection fraction, mean arterial blood pressure, and width of QRS [7]. Based on HFSS patients are classified into low ( $>8.10)$, medium (7.20-8.10) and high-risk $(<7.19)$ groups. The Seattle Heart Failure is another widely used prognostic model, 
which takes 5 out of 7 HFSS parameters (without heart rate and peak $\mathrm{VO}_{2}$ ), along with demographic parameters, use of devices and some laboratory data [8]. Direct comparison of these two models shows relatively good concordance, although the Seattle Heart Failure Model tends to be overly optimistic and HFSS more pessimistic in estimating survival $[9,10]$.

In our study, we analyzed 21 parameters but only age, ejection fraction [\%], SBP, DBP, LV end-systolic diameter, sodium serum level, HFSS and NT-proBNP, hs-CRP, PCWP, and PASP/SBP score influenced patients' survival in univariate Cox regression analysis.

Pulmonary hypertension may result from an increase in LV filling pressure, left atrial pressure, and PVR. Most data underlined strong prognostic value of PCWP and PH [11].

Even though most of patients qualified to HTx have pulmonary artery hypertension, some authors underline neutral influence of $\mathrm{PH}$ on their prognosis $[12,13]$. A possible explanation for this observation is that patients on the waiting list to HTx are highly selected, homogenous with terminal HF, thus limiting the chances for identifying differences. Finally, some patients with fixed PH are disqualified from HTx. Usually PH can cause right ventricular (RV) dysfunction. PH and RV dysfunction carry a poor prognosis and increase the risk of postoperative RV failure after HTx [14]. Tricuspid regurgitation is often a consequence of $\mathrm{PH}$. $\mathrm{RV}$ dysfunction is usually associated with reduced $\mathrm{CO}$ and pulmonary blood flow, so hemodynamic parameters of pulmonary circulation, especially prognostic value of PASP may be underestimated in these circumstances. Nevertheless, PASP exceeding $45 \mathrm{~mm} \mathrm{Hg}$ is associated with worse prognosis. Our results are in accordance with these observations.

Among 9 hemodynamic parameters analyzed only SBP, PCWP, and PASP/SBP score statistically influenced survival using univariate as well as multivariate Cox regression models.

Using Kaplan-Maier long rang test we compared probability of survival patients stratified by terciles. Pulmonary capillary wedge pressure above $25 \mathrm{~mm} \mathrm{Hg}$ was connected with significantly worse prognosis. One-year survival was 65\%, 3-year - 40\%. Patients with PCWP $<25 \mathrm{~mm} \mathrm{Hg}$ had 1-year and 3-year survival (80\% and $50 \%$, respectively). Pulmonary pressure has also significant influence on survival. Patients with PASP above $50 \mathrm{~mm} \mathrm{Hg}$ had worse prognosis compared to patients with PASP $<35 \mathrm{~mm} \mathrm{Hg}$. An interesting finding is that PASP exceeding $35 \mathrm{~mm} \mathrm{Hg}$ had negative influence on survival during first 200 days of observation as compared to patients with PASP $<35 \mathrm{~mm} \mathrm{Hg}$.

Score PASP/SBB higher than 0.52 had significant influence on survival in cardiac transplant candidates.

\section{Conclusions}

In patients with acute chronic HF qualified to HTx, pathological values of pulmonary hemodynamic parameters have a significant influence on survival. Any kind of pulmonary artery hypertension may influence short-term prognosis. Pulmonary hemodynamic parameters should be taken into account during allocation of ambulatory candidates for HTx.

\section{Acknowledgements}

Realized as a grant of the National Ministry of Health POLKARD 1304/IK-AG-K283/03 Clinical trials.gov. no NCT00690157.

POLKARD HF Investigators: Institute of Cardiology, Warsaw: Jerzy Korewicki, Aldona Browarek, Tomasz Zieliński, Małgorzata Sobieszczańska-Małek, Małgorzata Piotrowska, Jacek Różański, Jerzy Wołczyk, Piotr Kołsut, Walerian Piotrowski

Silesian Center for Heart Disease, Zabrze: Marian Zembala, Lech Poloński, Michał Zakliczyński, Piotr Rozentryt, Jolanta Nowak, Tomasz Kukulski, Marcin Świerad, Anna Barańska-Kosakowska Department of Heart, Vascular and Transplantology Surgery, Krakow: Jerzy Sadowski, Piotr Przybyłowski, Dariusz Sobczyk, Karol Wierzbicki

\section{Conflict of interest: None declared}

\section{References}

1. Grigioni F, Potena L, Galie N et al. Prognostic implications of serial assessments of pulmonary hypertension in severe chronic heart failure. J Heart Lung Transplant, 2006; 25: 1241-1246.

2. Ortiz V, Martinez-Dolz L, Sanchez-Lacuesta E et al. Clinical and hemodynamic profile of patients with advanced heart failure considered for heart transplantation. Transplant Proc, 2007; 39: 2341-2343.

3. Galie N, Hoeper M, Humbert M et al. Guidelines for the diagnosis and treatment of pulmonary hypertension. The Task Force for the Diagnosis and Treatment of Pulmonary Hypertension of the European Society of Cardiology (ESC) and the European Respiratory Society (ERS), endorsed by the International Society of Heart and Lung Transplantation (ISHLT). Eur Heart J, 2009; 30: 2493-2537.

4. Mehra M, Kobashigava J, Starling R et al. Listing criteria for transplantation: International Society for Heart and Lung Transplantation Guidelines for the care of cardiac transplant candidates 2006. J Heart Lung Transplant, 2006; 25: 1024-1042. 
Cardiology Journal 2014, Vol. 21, No. 5

5. Chang PP, Longenecker JC, Wang NY et al. Mild vs. severe pulmonary hypertension before heart transplantation: different effects on post transplantation pulmonary hypertension and mortality. J Heart Lung Transplant, 2005; 24: 998-1007.

6. Zieliński T, Kurjata P, Korewicki J. Prognosis in patients with severe heart failure referred for heart transplantation: POLKARD-HF 2003-2007. Int J Cardiol, 2010; 145: 242-244.

7. Mancini DM, Eisen H, Kussmal W et al. Value of peak exercise oxygen consumption for optimal timing of cardiac transplantation in ambulatory patients with heart failure. Circulation, 1991; 83: 778-786.

8. Levy WC, Mozaffarian D, Linker DT et al. The Seattle Heart Failure Model prediction of survival in heart failure. Circulation, 2006; 113: 1424-1443.

9. Aaronson KD, Schwartz JS, Chen TM et al. Development and prospective validation of a clinical index to predict survival in ambulatory patients referred for cardiac transplant evaluation. Circulation, 1997; 95: 2660-2667.
10. Mancini D, Lietz K. Selection of cardiac transplantation candidates in 2010. Circulation, 2010; 122: 173-183.

11. Chen J, Levin H, Michler R et al. Revaluating the significance of pulmonary hypertension before cardiac transplantation: determination of optimal thresholds and quantification of the effect of reversibility on perioperative mortality. J Thorac Cardiovasc Surg, 1997; 114: 627-634.

12. Damy T, Goode K, Kallvikbacka-Bennet A et al. Determinants and prognostic value of pulmonary arterial pressure in patients with chronic heart failure. Eur Heart J, 2010; 31: 2280-2290.

13. Kjaergaard J, Akkan D, Karmark Iverson K et al. Prognostic importance of pulmonary hypertension in patients with heart failure. Am J Cardiol, 2007; 99: 1146-1150.

14. Chang P, Longenecker J, Wang N et al. Mild vs. severe pulmonary hypertension before heart transplantation: different effects on posttransplantation pulmonary hypertension and mortality. J Heart Lung Transplant, 2005; 24: 998. 